\title{
Thermo-Elastic Analysis of Clamped-Clamped Thick FGM Cylinders by Using Third-Order Shear Deformation Theory
}

\section{Abstract}

Using the third-order shear deformation theory (TSDT), an analytical solution for deformations and stresses of axisymmetric clamped-clamped thick cylindrical shells made of functionally graded material (FGM) subjected to internal pressure and thermal loading are presented. The material properties are graded along the radial direction according to power functions of the radial direction. It is assumed that Poisson's ratio is constant across the cylinder thickness. The differential equations governing were generally derived, making use of TSDT. Following that, the set of non-homogenous linear differential equations for the cylinder with clamped-clamped ends was solved, and the effect of loading and supports on the stresses and displacements was investigated. The problem was also solved, using the finite element method (FEM), and the results of which were compared with those of the analytical method. Furthermore, the effect of increases in the temperature gradient on displacement and stress values has been studied. Finally, in order to investigate the effect of third-order approximations on displacements and stresses, a comparison between the results of first- and third-order shear deformation theory has been made.

\section{Keywords}

Thick cylinder, Clamped-clamped, Thermo-elastic; Third-order shear deformation theory (TSDT), Functionally graded material (FGM).
H. Gharooni ${ }^{\text {a,* }}$

M. Ghannad ${ }^{\text {a }}$

M.Z. Nejad ${ }^{b}$

a Faculty of Mechanical Engineering,

Shahrood University of Technology,

Shahrood, Iran.

b Department of Mechanical Engineering, Yasouj University, Yasouj, Iran.

Corresponding author:

Gharooni.hamed@gmail.com

http://dx.doi.org/10.1590/1679-78252254

Received 27.06.2015

In revised form 03.12.2015

Accepted 11.01.2016

Available online 17.02.2016

\section{INTRODUCTION}

In the 1980's, functionally graded materials (FGMs) were alternative materials initially used in ospace structural applications and fusion reactors because of their particular material properties which vary smoothly and continuously from one surface to the another (Yamanoushi et al. 1990; Koizumi 1993). FGMs represent a new generation of composite materials whose microstructure ies from one material to another with a specific gradient. This smooth variation of material proper- 
ties significantly improves the mechanical strength and fracture toughness of FGMs (Cao et al. 2012). Since thick cylindrical shells such as hollow cylinders are used in various industrial fields as structure materials, thermo-elastic analysis of these structures under mechanical and thermal loads, which may cause undesirable stress and deformation, has become important. In order to reduce these stresses and deformations, hollow cylindrical shells made up of FGMs have been widely used. Most of the existing literature deals with thermo-elastic analysis of FG shells.

Reddy and Liu (1985) developed a simple third-order shear deformation shell theory, in which the transverse shear strains are assumed to be parabolically distributed across the shell thickness and which contains the same number of dependent unknowns as in the first-order shear deformation theory. Moreover, this theory requires no shear correction factors. Fukui and Yamanaka (1992) the Navier solution to derive the governing equation for a thick-walled FGM tube under internal pressure and solved the equations obtained numerically by means of the Runge-Kutta method. Eipakchi et al. (2003) investigated the governing equations of homogeneous cylinders with variable thickness, using FSDT and presented the solution of the equations, using perturbation theory. They further extended their previous work by considering homogenous and isotropic conical shells with variable thickness, using FSDT and SSDT (second-order shear deformation theory) and solved the conducted equations by perturbation theory (Eipakchi et al. 2008). Xiang et al. (2006) obtained the exact solution of FGM hollow cylinders in the state of plane strain with exponential function of elasticity modulus along the radius. Shi et al. (2007) analyzed heterogeneous cylindrical shells with power function of elasticity modulus by using multilayer method with homogeneous layers. Thickwalled FGM cylinders in plane strain state with exponentially-varying material properties were solved by Tutuncu (2007) using Frobenius method. Shao and Ma (2008) solved the governing difential equations of functionally graded circular hollow cylinders with exponential variation of material properties under mechanical loads and linearly increasing boundary temperature, using series expansion. Nejad, Rahimi, and Ghannad (2009) developed a 3-D set of field equations of FGM thick shells of revolution in curvilinear coordinate system by tensor calculus. Ghannad and Nejad (2010) presented the general method of derivation and the analysis of internally pressurized thick-walled cylinders with clamped-clamped ends. Eipakchi (2010) calculated stresses and displacements of a thick conical shell with varying thickness under non-uniform internal pressure analytically, using third-order shear deformation theory. Arefi and Rahimi (2010) conducted thermo-elastic analysis of a functionally graded cylinder under internal pressure, using first-order shear deformation theory for plane strain condition. Arani et al. (2011) investigated the effect of material inhomogeneity on electro-thermo-mechanical behaviors of FG piezoelectric rotating cylinders under internal and external pressure with varying properties of power function. Keles and Conker (2011) indicated transient hyperbolic heat condition in thick-walled FGM cylinders and spheres with exponentially-varying properties. Jabbari et al. (2011) indicated mechanical and thermal stresses in an functionally graded porous material (FGPM) hollow cylinder subjected to radially symmetric thermal loads and solved the governing equations by using Fourier series. Ghannad et al. (2012) presented a closed-form analytical solution for clamped-clamped thick cylindrical shells with variable thickness subjected to constant internal pressure based on the first-order shear deformation theory (FSDT). Ghannad et al. (2013) investigated the elastic analysis of pressurized thick cylindrical shells with variable thickness made of functionally graded materials. They used the matched asymptotic method (MAM) of 
the perturbation theory in order to convert the resultant equations into a system of algebraic equations and two systems of differential equations with constant coefficients. Three dimensional free vibration and transient response of a cylindrical panel made of two directional functionally graded materials based on three dimensional equations of elasticity and subjected to internal impact loading is considered by Zafarmand et al. (2015). Nejad et al. (2015) presented a semi-analytical solution for the purpose of elastic analysis of rotating thick truncated conical shells made of FGM under non-uniform pressure by using the first-order shear deformation theory (FSDT) and multilayer method (MLM). Ghannad and Gharooni (2015) investigated displacements and stresses in pressurized thick FGM cylinders with exponential variation of material properties based on TSDT.

Most of the studies reviewed have investigated the heat transfer and gradient temperature of FGM cylinders and have presented no accurate solution especially the ones which use shear deformation theory. The results of previous analyses of the cylindrical shells show that FSDT is not an appropriate theory for the purpose of stress analysis, suggesting that the accuracy of the stresses (especially radial stresses), unlike displacements which have been calculated directly by SDT, are not acceptable. Furthermore, it could be seen that the thermo-elastic analysis of pressurized thick FGM cylinders based on third-order shear deformation theory has not been studied in the literature. On the other hand, other theories such as classical theories or plane elasticity theories (PET) have not acceptable results because of failing to take into account shear stresses.

In order to improve the approximation of shear deformation theory (especially under thermal loading) and to take into account the effect of shear stresses and strains, the general method of derivation and thermo-elastic analysis of pressurized thick-walled cylindrical shells under the effect of gradient temperature has been presented in this paper by using HSDT. The material of the cylinder is assumed to be isotropic and heterogeneous with radially varying mechanical and physical properties continuously along the thickness with a power function and constant Poisson's ratio. The coefficient matrices of the governing equations have been derived in the unique abbreviated form. The obtained equations are solved under the generalized clamped-clamped conditions. Furthermore, the effects of loading, temperature gradient and inhomogeneity of FG materials on the stresses and displacements have been investigated. The analytical results of shear deformation theory are compared with the numerical results of FEM. Finally, the higher efficiency and accuracy of the thirdorder shear deformation theory, compared with the lower one, have been shown.

\section{PROBLEM FORMULATION}

In shear deformation theory (SDT), the straight lines perpendicular to the central axis of the cylinder do not necessarily remain unchanged after loading and deformation, suggesting that the deformations are axisymmetric and change along the longitudinal direction of cylinder. This means that the elements have rotation, and the shear strain is not zero.

The parameter $r$ is the radius of every layer of cylinder which can be replaced in terms of radius of mid-plane $(R)$ and distance of every layer with respect to mid-plane $(z)$, as follows (Figure 1):

$$
r=R+Z
$$


The parameters $x$ and $z$ are the length and the thickness variables which vary in the following intervals:

$$
0 \leq x \leq L \quad,-\frac{h}{2} \leq z \leq+\frac{h}{2}
$$

where $h$ and $L$ are the thickness and the length of the cylinder.

The displacement field is assumed as a polynomial of a variable $(z)$ through the thickness. As the number of terms in the polynomial function increases, the approximate solution is improved as well. The high-order shear deformation theory (HSDT) is employed to simulate the deformation of every layer of the cylinder.

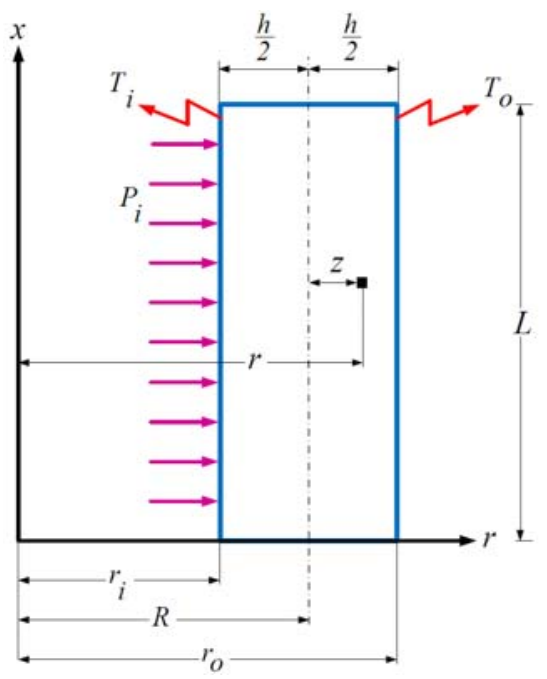

Figure 1: Geometry of the thick cylindrical shell under internal pressure and temperature gradient.

Based on HSDT, every component of deformation can be stated by variables that include the displacement and rotation. For an axisymmetric cylindrical shell, axial and radial components of displacement field are assumed to be in the following form:

$$
\left\{\begin{array}{l}
U_{x}=u_{0}(x)+z u_{1}(x)+z^{2} u_{2}(x)+z^{3} u_{3}(x) \\
U_{\theta}=0 \\
U_{z}=w_{0}(x)+z w_{1}(x)+z^{2} w_{2}(x)+z^{3} w_{3}(x)
\end{array}\right.
$$

where $u_{0}(x)$ and $w_{0}(x)$ are the displacement components of the middle surface. $u_{i}(x)$ and $w_{i}(x)$ for $i=0,1,2,3$ are the unknown functions of $x$ which are used to determine the displacement field.

The kinematic relations in the cylindrical coordinates system for an axisymmetric cylinder are: 


$$
\left\{\begin{array}{l}
\varepsilon_{x}=\frac{\partial U_{x}}{\partial x}=\frac{d u_{0}}{d x}+\frac{d u_{1}}{d x} z+\frac{d u_{2}}{d x} z^{2}+\frac{d u_{3}}{d x} z^{3} \\
\varepsilon_{\theta}=\frac{U_{z}}{r}=\frac{w_{0}+w_{1} z+w_{2} z^{2}+w_{3} z^{3}}{R+z} \\
\varepsilon_{z}=\frac{\partial U_{z}}{\partial z}=w_{1}+2 w_{2} z+3 w_{3} z^{2} \\
\gamma_{x z}=\frac{\partial U_{x}}{\partial z}+\frac{\partial U_{z}}{\partial x}=\left(u_{1}+\frac{d w_{0}}{d x}\right)+\left(2 u_{2}+\frac{d w_{1}}{d x}\right) z+\left(3 u_{3}+\frac{d w_{2}}{d x}\right) z^{2}+\frac{d w_{3}}{d x} z^{3}
\end{array}\right.
$$

Considering the dimensionless radial coordinate $(\bar{r})$ as the ratio of radial coordinate $(r)$ upon internal radius $\left(r_{i}\right)$ :

$$
\bar{r}=\frac{r}{r_{i}}
$$

Mechanical and physical properties of inhomogenous and isotropic material (FGM) including modulus of elasticity $(E)$, thermal expansion coefficient $(\alpha)$ and thermal conductivity coefficient $(K)$ are supposed to be a power function of dimensionless radial coordinate:

$$
\begin{aligned}
& E(r)=E_{i}(\bar{r})^{n} \\
& \alpha(r)=\alpha_{i}(\bar{r})^{n} \\
& K(r)=K_{i}(\bar{r})^{n}
\end{aligned}
$$

Here $E_{i}, \alpha_{i}$ and $K_{i}$ are the modulus of elasticity, thermal expansion coefficient and thermal conductivity coefficient at the inner surface $\left(r_{i}\right)$ of the cylinder and $n$ is the inhomogeneity constant of FG material determined empirically. Generally speaking, the Poisson's ratio $(v)$ for a thickwalled cylindrical pressure vessel of isotropic FGM varies in a small range. Furthermore, its effects on mechanical stresses are insignificant. For simplicity, the Poisson's ratio is assumed to be constant.

The distribution of material properties from Eqs. (6) can be re-written as a function of $z$ by substituting $r$ from Eq. (1) into Eq. (5):

$$
\begin{aligned}
& E(z)=E_{i}\left(\frac{R+z}{r_{i}}\right)^{n} \\
& \alpha(z)=\alpha_{i}\left(\frac{R+z}{r_{i}}\right)^{n}
\end{aligned}
$$




$$
K(z)=K_{i}\left(\frac{R+z}{r_{i}}\right)^{n}
$$

Figure 2 shows the distribution of dimensionless material properties $\left(x / x_{i}\right)$ with respect to the dimensionless radius in a heterogeneous cylinder for integer values of $n$ which vary in the range of $-2 \leq n \leq+2$.

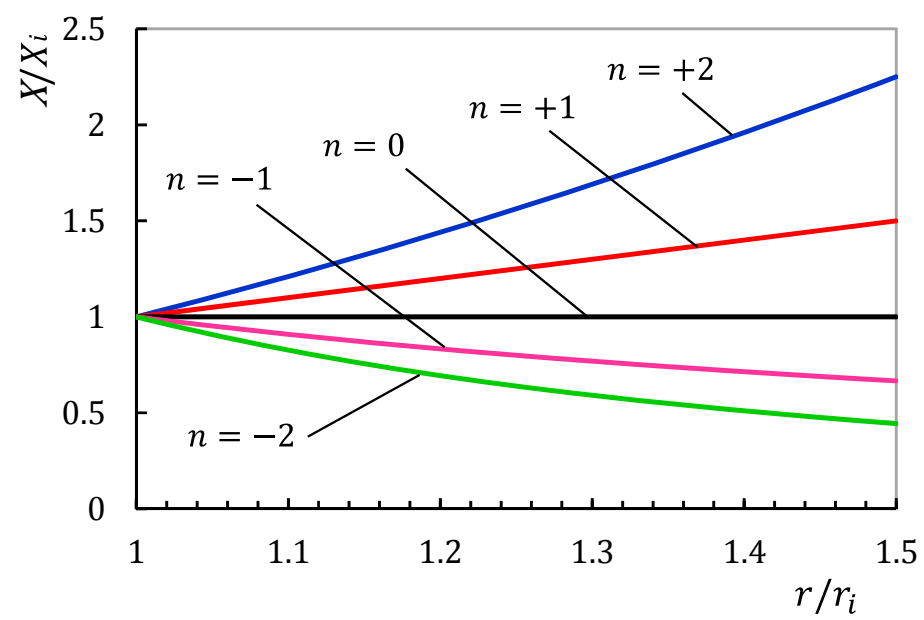

Figure 2: Distribution of dimensionless material properties in an FGM cylinder.

On the basis of the constitutive equations for inhomogeneous and isotropic materials, the stress components based on components of mechanical and thermal strain are as follows:

$$
\left\{\begin{array}{c}
\sigma_{x} \\
\sigma_{\theta} \\
\sigma_{z} \\
\tau_{x \theta} \\
\tau_{\theta z} \\
\tau_{x z}
\end{array}\right\}=\left[\begin{array}{cccccc}
\lambda+2 \mu & \lambda & \lambda & 0 & 0 & 0 \\
\lambda & \lambda+2 \mu & \lambda & 0 & 0 & 0 \\
\lambda & \lambda & \lambda+2 \mu & 0 & 0 & 0 \\
0 & 0 & 0 & 0 & 0 & 0 \\
0 & 0 & 0 & 0 & 0 & 0 \\
0 & 0 & 0 & 0 & 0 & \mu
\end{array}\right]\left\{\begin{array}{c}
\varepsilon_{x} \\
\varepsilon_{\theta} \\
\varepsilon_{z} \\
\gamma_{x \theta} \\
\gamma_{\theta z} \\
\gamma_{x z}
\end{array}\right\}-\frac{\alpha(z) E(z)}{1-2 v}\left\{\begin{array}{c}
\Delta T(z) \\
\Delta T(z) \\
\Delta T(z) \\
0 \\
0 \\
0
\end{array}\right\}
$$

where $\lambda$ and $\mu$ are the Lame's constants. Considering variable elasticity modulus for the FGM materials, these two parameters are as follows:

$$
\begin{gathered}
\lambda=\frac{v E(z)}{(1+v)(1-2 v)} \\
\mu=\frac{E(z)}{2(1+v)}
\end{gathered}
$$

Furthermore, $\Delta T(\mathrm{z})$ is the distribution of temperature gradient along the thickness of the cylinder in the one-dimensional steady state heat transfer condition. 
The axial forces based on normal components of stress are as follows:

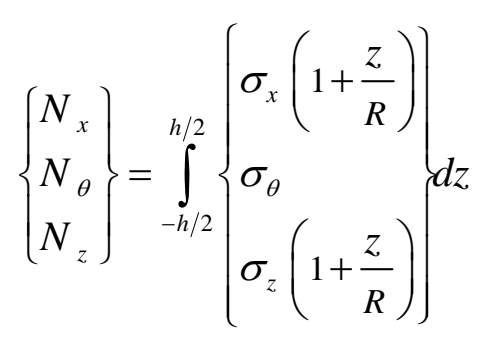

The bending moments based on normal components of stress are as follows:

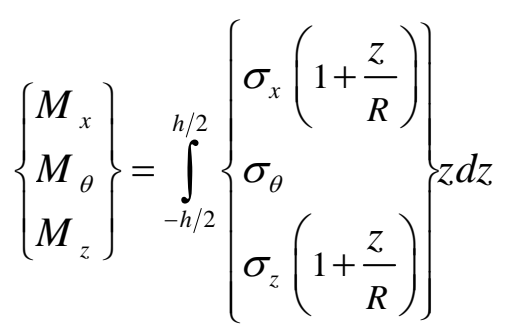

The higher-order bending moments based on normal components of stress are as follows:

$$
\begin{aligned}
& \left\{\begin{array}{l}
P_{x} \\
P_{\theta} \\
P_{z}
\end{array}\right\}=\int_{-h / 2}^{h / 2}\left\{\begin{array}{l}
\sigma_{x}\left(1+\frac{z}{R}\right) \\
\sigma_{\theta}\left(1+\frac{z}{R}\right) \\
\sigma_{z}(1)
\end{array}\right\} z^{2} d z \\
& \left\{\begin{array}{l}
S_{x} \\
S_{\theta}
\end{array}\right\}=\int_{-h / 2}^{h / 2}\left\{\sigma_{x}\left(1+\frac{z}{R}\right)\right\} z^{3} d z
\end{aligned}
$$

The shear force based on shear stress is as follows:

$$
Q_{x}=\int_{-h / 2}^{h / 2} \tau_{x z}\left(1+\frac{z}{R}\right) d z
$$

The torsional moment based on shear stress is as follows:

$$
M_{x z}=\int_{-h / 2}^{h / 2} \tau_{x z}\left(1+\frac{Z}{R}\right) z d z
$$

The higher-order torsional moments based on shear stress are as follows: 


$$
\begin{aligned}
& P_{x z}=\int_{-h / 2}^{h / 2} \tau_{x z}\left(1+\frac{z}{R}\right) z^{2} d z \\
& S_{x z}=\int_{-h / 2}^{h / 2} \tau_{x z}\left(1+\frac{z}{R}\right) z^{3} d z
\end{aligned}
$$

\section{VIRTUAL WORK PRINCIPLE}

Based on the principle of virtual work, the variation of strain energy of the elastic body $(U)$ is equal to the variation of external work due to pressure $(W)$.

$$
\delta U=\delta W
$$

The strain energy is

$$
\left\{\begin{array}{l}
U=\iiint_{V} U^{*} d V, d V=r d r d \theta d x=(R+z) d x d \theta d z \\
U^{*}=\frac{1}{2}\{\varepsilon\}^{T}\{\sigma\}=\frac{1}{2}\left(\sigma_{x} \varepsilon_{x}+\sigma_{\theta} \varepsilon_{\theta}+\sigma_{z} \varepsilon_{z}+\tau_{x z} \gamma_{x z}\right)
\end{array}\right.
$$

and the external work consists of internal and external pressure $\left(P_{i}\right.$ and $\left.P_{o}\right)$, which is:

$$
\left\{\begin{array}{l}
W=\iint_{S}\left(\overrightarrow{f_{s f}} \cdot \vec{u}\right) d S, d S=r d \theta d x \\
\left(\overrightarrow{f_{s f}} \cdot \vec{u}\right) d S=\left(P_{i} r_{i}-P_{o} r_{o}\right) U_{z} d \theta d x
\end{array}\right.
$$

where $\overrightarrow{f_{s f}}$ is the surface force of the pressurized cylinder.

The bounds of integrals for $X$ and $Z$ variables have been defined in Eq. (2).

Variation of the strain energy can be expressed as follows:

$$
\left\{\begin{array}{l}
\delta U=R \int_{0}^{2 \pi} \int_{0}^{L} \int_{h / 2}^{h / 2} \delta U^{*}\left(1+\frac{Z}{R}\right) d z d x d \theta \\
\Rightarrow \frac{\delta U}{2 \pi}=R \int_{0}^{L} \int_{-h / 2}^{h / 2}\left(\sigma_{x} \delta \varepsilon_{x}+\sigma_{\theta} \delta \varepsilon_{\theta}+\sigma_{z} \delta \varepsilon_{z}+\tau_{x z} \delta \gamma_{x z}\right)\left(1+\frac{Z}{R}\right) d z d x
\end{array}\right.
$$

and the variation of the external work is:

$$
\left\{\begin{array}{l}
\delta W=\int_{0}^{2 \pi} \int_{0}^{L}\left[P_{i} r_{i}-P_{o} r_{o}\right] \delta U_{z} d x d \theta \\
\Rightarrow \frac{\delta W}{2 \pi}=\int_{0}^{L}\left[P_{i}\left(R-\frac{h}{2}\right)-P_{o}\left(R+\frac{h}{2}\right)\right] \delta U_{z} d x
\end{array}\right.
$$


Substituting Eqs. (4), (7) and (8) into Eqs. (22) and (23), using Eq. (19) and carrying out the integration by parts, the equilibrium equations for the cylindrical shell with constant thickness under one-dimensional heat transfer and uniform internal and external pressure are obtained in the form of:

$$
\left\{\begin{array}{l}
R \frac{d N_{x}}{d x}=F_{x_{0}}^{P}+F_{x_{0}}^{T} \\
R \frac{d M_{x}}{d x}-R Q_{x}=F_{x_{1}}^{P}+F_{x_{1}}^{T} \\
R \frac{d P_{x}}{d x}-2 R M_{x z}=F_{x_{2}}^{P}+F_{x_{2}}^{T} \\
R \frac{d S_{x}}{d x}-3 R P_{x z}=F_{x_{3}}^{P}+F_{x_{3}}^{T} \\
R \frac{d Q_{x}}{d x}-N_{\theta}=F_{z_{0}}^{P}+F_{z_{0}}^{T} \\
R \frac{d M_{x z}}{d x}-M_{\theta}-R N_{z}=F_{z_{1}}^{P}+F_{z_{1}}^{T} \\
R \frac{d P_{x z}}{d x}-P_{\theta}-2 R M_{z}=F_{z_{2}}^{P}+F_{z_{2}}^{T} \\
R \frac{d S_{x z}}{d x}-S_{\theta}-3 R P_{z}=F_{z_{3}}^{P}+F_{z_{3}}^{T}
\end{array}\right.
$$

where $F^{P}$ and $F^{T}$ stand for non-homogeneity of the governing equations which have resulted from the loading of pressure and temperature, respectively. The subscripts $x$ and $z$ in the right terms of each equation show the components of $F^{P}$ and $F^{T}$ along the axial and radial direction, respectively.

$$
\begin{gathered}
\left\{\begin{array}{l}
F_{x_{i}}^{P}=0 \\
F_{z_{i}}^{P}=-\left.R f_{s f} z^{i}\left(1+\frac{z}{R}\right)\right|_{z= \pm \frac{h}{2}} i=0,1,2,3
\end{array}\right. \\
\left\{\begin{array}{l}
F_{x_{i}}^{T}=0 \\
F_{z_{i}}^{T}=-\int_{-h / 2}^{h / 2} \frac{\alpha(z) E(z) \Delta T(z)}{1-2 v}\left((i+1) z^{i}+i R z^{i-1}\right) d z \quad i=0,1,2,3
\end{array}\right.
\end{gathered}
$$

The boundary conditions at the two ends of the cylinder are:

$$
R\left[N_{x} \delta u_{0}+M_{x} \delta u_{1}+P_{x} \delta u_{2}+S_{x} \delta u_{3}+Q_{x} \delta w_{0}+M_{x z} \delta w_{1}+P_{x z} \delta w_{2}+S_{x z} \delta w_{3}\right]_{0}^{L}=0
$$

Eqs. (24) express the main governing equations based on the HSDT for the cylindrical shells under mechanical and thermal loading. Eq. (27) is the boundary conditions which should be satisfied at the two ends of the cylinder. 


\section{HEAT TRANSFER EQUATION}

According to Figure 1, heat transfer in the form of conduction along the thickness of the cylinder under the effect of heat sources at the internal and external surface causes thermal strains. For thermo-elastic analysis of the cylinder, the function of temperature gradient distribution along the longitudinal direction should be determined by the solution of heat transfer equation. Fourier's heat transfer law in general form is:

$$
\nabla^{2}(K(r) T)+\dot{q}=\rho C \rho^{T}
$$

where $T, K(r), \dot{q}, \rho$ and $C_{\rho}$ are temperature distribution, thermal conduction coefficient, heat generation rate, density and specific heat, respectively.

In cylindrical coordinate system, the Eq. (28) could be re-written in the form of:

$$
\frac{1}{r} \frac{\partial}{\partial r}\left(K(r) r \frac{\partial T}{\partial r}\right)+\frac{1}{r^{2}} \frac{\partial}{\partial \theta}\left(K(r) \frac{\partial T}{\partial r}\right)+\frac{\partial}{\partial x}\left(K(r) \frac{\partial T}{\partial x}\right)+\dot{q}=\rho C_{p}{ }^{T}
$$

Axisymmetric thermal load in cylindrical coordinate system causes just radial flow in the cylinder. Therefore, Eq. (29) for radial temperature distribution in steady-state condition without any heat generation has been simplified as:

$$
\frac{\partial}{\partial r}\left(K(r) r \frac{\partial T}{\partial r}\right)=0
$$

Thermal boundary conditions, as shown in Figure 1, consist of temperatures at the internal and external surfaces of the cylinder:

$$
\left.T(r)\right|_{r=r_{i}}=T_{i},\left.T(r)\right|_{r=r_{o}}=T_{o}
$$

Solution of differential Eq. (30) by applying thermal boundary conditions according to Eq. (31) and considering coefficients of thermal conduction from Eqs. (6- c) yield to the temperature distribution along the thickness of the cylinder as a function of radius:

$$
T(r)=\frac{\left(r_{i} r_{o}\right)^{n}}{\left(r_{i}^{n}-r_{o}^{n}\right)}\left[\left(\frac{T_{o}-T_{i}}{r^{n}}\right)-\left(\frac{T_{o}}{r_{i}^{n}}\right)+\left(\frac{T_{i}}{r_{o}^{n}}\right)\right]
$$

Since the terms of temperature gradient distribution appear in the thermal part of nonhomogeneity in Eq. (26) $\left(F^{T}\right)$, Eq. (32) should be re-written in the terms of $\Delta T(z)$ :

$$
\Delta T(z)=\frac{\left(r_{i} r_{o}\right)^{n}}{\left(r_{i}^{n}-r_{o}^{n}\right)}\left[\left(\frac{\Delta T_{o}-\Delta T_{i}}{(R+z)^{n}}\right)-\left(\frac{\Delta T_{o}}{r_{i}^{n}}\right)+\left(\frac{\Delta T_{i}}{r_{o}^{n}}\right)\right]
$$


where $\Delta T_{i}$ and $\Delta T_{o}$ are the temperature gradients between the environment of the cylinder and internal and external surfaces, respectively. Considering $T_{r e f}$ as the temperature of environment (reference), we have:

$$
\Delta T_{i}=T_{i}-T_{\text {ref }}, \quad \Delta T_{o}=T_{o}-T_{\text {ref }}
$$

\section{ANALYTICAL SOLUTION}

Eqs. (24) are the set of differential equations. In order to solve these equations, forces and moments could be written by using Eqs. (11) to (18) in terms of stresses. The stresses could be written in terms of strains by substituting Eqs. (7- a and b) into Eq. (8) and using Eq. (33). By using Eqs. (4), the strains are converted into the displacement filed components. Finally, a set of linear nonhomogenous differential equations with constant coefficients would result, as follows:

$$
[A] \frac{d^{2}}{d x^{2}}\{y\}+[B] \frac{d}{d x}\{y\}+[C]\{y\}=\{F\}
$$

where $[A]_{8 \times 8},[B]_{8 \times 8}$ and $[C]_{8 \times 8}$ are the coefficient matrices and $\{F\}$ is the force vector, which can be expressed as the set of non-homogeneity of differential equations'. $\{y\}$ is the unknown vector including the components of displacement field as:

$$
\{y\}=\left\{\begin{array}{llllllll}
u_{0} & u_{1} & u_{2} & u_{3} & w_{0} & w_{1} & w_{2} & w_{3}
\end{array}\right\}^{T}
$$

Matrix $[C]$ in the Eq. (35) whose reverse would be needed in the next calculations is irreversible. In order to make $[C]^{-1}$, the first equation in the set of Eqs. (24) has been integrated.

$$
R N_{x}=C_{0}
$$

In Eqs. (24), it is apparent that $u_{0}$ does not exist, but $d u_{0} / d x$ does. In order to calculate deformations in Eqs. (4), $d u_{0} / d x$ is needed. Therefore, by assuming $d u_{0} / d x=v$ as a new parameter which could be indicated in the following terms, we have:

$$
u_{0}=\int v d x+C_{15}
$$

Applying the mentioned changes, the unknown vector $\{y\}$ in the set of differential Eqs. (24) would be rewritten as follows:

$$
\{y\}=\left\{\begin{array}{llllllll}
v & u_{1} & u_{2} & u_{3} & w_{0} & w_{1} & w_{2} & w_{3}
\end{array}\right\}^{T}
$$

Non-homogeneity of the differential Eqs. (24) would be derived as follows: 


$$
\{F\}=\left\{\begin{array}{c}
F_{x_{0}}^{P}+F_{x_{0}}^{T}+C_{0} \\
F_{x_{1}}^{P}+F_{x_{1}}^{T} \\
F_{x_{2}}^{P}+F_{x_{2}}^{T} \\
F_{x_{3}}^{P}+F_{x_{3}}^{T} \\
F_{z_{0}}^{P}+F_{z_{0}}^{T} \\
F_{z_{1}}^{P}+F_{z_{1}}^{T} \\
F_{z_{2}}^{P}+F_{z_{2}}^{T} \\
F_{z_{3}}^{P}+F_{z_{3}}^{T}
\end{array}\right\}
$$

The corresponding coefficient matrices $[A],[B]$ and $[C]$ of the new differential Eqs. (35) have been defined in appendices.

The solution of Eqs. (35) consists of general and particular parts:

$$
\{y\}=\{y\}_{g}+\{y\}_{p}
$$

For the general solution, $\{y\}_{g}=\{V\} e^{m x}$ is substituted in homogeneous Eq. (26).

$$
e^{m x}\left[m^{2}[A]+m[B]+[C]\right]\{V\}=\{0\}
$$

Considering that $e^{m x}$ is not equal to zero, the following determinant which is equal to zero would result.

$$
\left|m^{2}[A]+m[B]+[C]\right|=0
$$

The above determinant is a sixteen-order polynomial which is a function of $m$. The determinant's roots are the eigenvalues $m_{i}$ consisting of eight pairs of conjugated roots where a pair of the roots is zero. Substituting the calculated eigenvalues in Eq. (42), the corresponding eigenvectors $\{V\}_{i}$ are obtained. Therefore, the general solution has been obtained.

$$
\{y\}_{g}=\sum_{i=1}^{14} C_{i}\{V\}_{i} e^{m_{i} x}
$$

Given that $\{F\}$ in Eq. (35) consists of constant parameters, the non-homogenous part of the solution for axisymmetric cylinder with constant thickness under uniform pressure is not the function of $x$. Therefore, the particular solution can be expressed as follows.

$$
[C]\{y\}_{p}=\{F\} \Rightarrow\{y\}_{p}=[C]^{-1}\{F\}
$$

Considering Eq. (27), clamped-clamped boundary conditions at two ends of the cylinder are as follows: 


$$
\left\{\begin{array}{l}
x=0 \Rightarrow u_{0}=u_{1}=u_{2}=u_{3}=w_{0}=w_{1}=w_{2}=w_{3}=0 \\
x=L \Rightarrow u_{0}=u_{1}=u_{2}=u_{3}=w_{0}=w_{1}=w_{2}=w_{3}=0
\end{array}\right.
$$

Applying eight boundary conditions at each end of the cylinder, one can calculate sixteen constants comprised of $C_{1}, \ldots, C_{14}$ in the general solution and $C_{0}, C_{15}$ in the particular solution. Finally, the unknown vector $\{y\}$ which consists of displacement field components would be obtained in terms of $X$ variable based on Eq. (41) by determining unknown constants. Using Eq. (3) would yield radial and axial displacements. Stress distribution would be obtained by using Eqs. (4) and (8).

\section{NUMERICAL RESULTS AND DISCUSSIONS}

As a case study, a thick heterogeneous cylinder under uniform internal pressure and heat conduction resulting from temperature gradient of internal and external surfaces and clamped-clamped boundary conditions at the two ends with the following characteristics could be considered: $r_{i}=40 \mathrm{~mm}, h=20 \mathrm{~mm}$ and $L=0.8 \mathrm{~m}$. The values of elasticity modulus, thermal expansion coefficient and thermal conductivity coefficient at the internal radius are $E_{i}=200 \mathrm{GPa}$, $\alpha_{i}=12 \times 10^{-6} /{ }^{\circ} \mathrm{C}$ and $K_{i}=20 \mathrm{~W} / \mathrm{m} . \mathrm{K}$, respectively. The value of Poisson's ratio is $v=0.3$. The applied internal pressure is $P_{i}=80 \mathrm{MPa}$. The temperatures of internal and external surfaces are $T_{i}=125{ }^{\circ} \mathrm{C}$ and $T_{o}=25{ }^{\circ} \mathrm{C}$, respectively. The environment (reference) temperature is $T_{\text {ref }}=25{ }^{\circ} \mathrm{C}$. The analytical solution has been carried out by writing the program in MAPLE 17.

In order to demonstrate the potentials of the presented analytical solution for the purpose of analyzing an FG cylinder, a numerical solution is investigated. The ANSYS 14.5 package is used in the static analysis of thick hollow cylinder with constant thickness. The PLANE82 element in the axisymmetric mode, which is an element with eight nodes and two translational degrees of freedom in the axial and radial directions per each node, has been used to model the mechanical part of the analysis. For the thermal part of the analysis, the appropriate element is PLANE77, which is compatible with initial element for superposing the thermal and mechanical results. The length and the thickness of the cylinder have been divided into 1600 and 40 parts, respectively. The quadratic mapped meshing has been applied for each section of the cylinder. In order to consider power-law varying elastic modulus along the thickness of the cylindrical shell, the thickness of the cylinder has been divided into some homogeneous layers. As applying more than 40 layers along the thickness shows no significant effect in FEM results, the thickness of the cylinder is divided into 40 homogeneous layers. Each layer's properties have been defined as a power function of the distance of layer's middle from the internal layer. Finally, the cylindrical shell consists of some coherent homogeneous layers. In order to merge the layers, the properties of contact location of the layers have been defined the average of left and right limits of the boundaries of the two adjacent layers. For thermal boundary conditions, the temperature gradient values of the inner and outer surfaces with the environment temperature have been applied to the corresponding nodes. Clamped boundary conditions 
have been exerted by preventing the nodes around the two ends of the cylinder from movement. In the next sections, the numerical and analytical results have been investigated.

\subsection{Loading and Inhomogeneity Effect}

The distribution of the dimensionless radial displacement resulting from the numerical and analytical solution in the middle of a cylinder under $100{ }^{\circ} \mathrm{C}$ temperature gradient is depicted in Figures 3 and 4 without and with internal pressure, respectively. It is evident that radial displacement under temperature gradient for negative values of $n$ is higher than the homogenous materials at layers close to the internal surface while at the outer surface, reverse holds true. For positive values of $n$, the reverse holds true, which means that the heterogonous materials have lower values of radial displacement than the homogenous ones at inner surfaces and the higher values of displacement at outer surfaces. The variation in the displacement of heterogeneous materials is similar to that of homogenous materials close to the middle layer. Under thermal and mechanical loads for negative values of $n$, the displacements of FGM cylinders are higher than those of the homogeneous cylinder. For positive values of $n$, the situation is reverse, i.e. the displacement is lower. Figure 4 shows that radial displacements for different values of $n$ increase from internal layer to the middle layer while in the external half of the cylinder's thickness, they decrease around the middle layer to the external layer. Variation of inhomogeneity constants from negative to the positive causes maximum displacement of cylinder's thickness to appear around the external layer. Furthermore, adding internal pressure to the temperature gradient causes uniform displacement along the radial direction. From the viewpoint of low level and small variation of displacements, using materials with negative inhomogeneity constants for thermal loading and positive for mechanical loading is recommended.

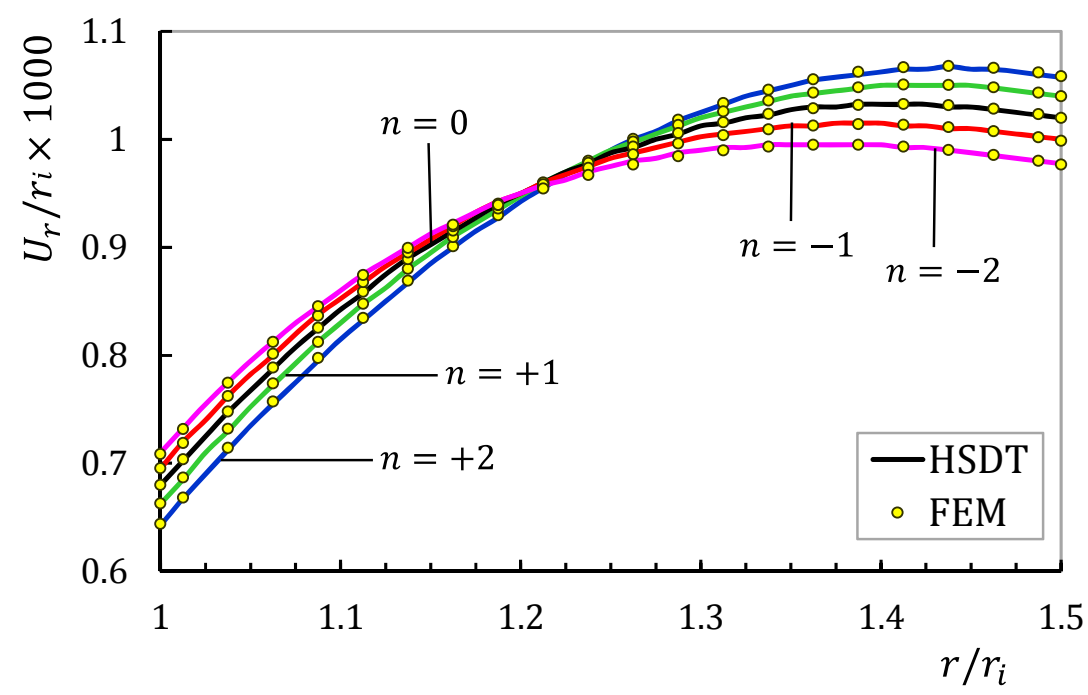

Figure 3: Dimensionless radial displacement distribution in the middle of the cylinder under temperature gradient $\left(\Delta T=100^{\circ} \mathrm{C}\right)$. 


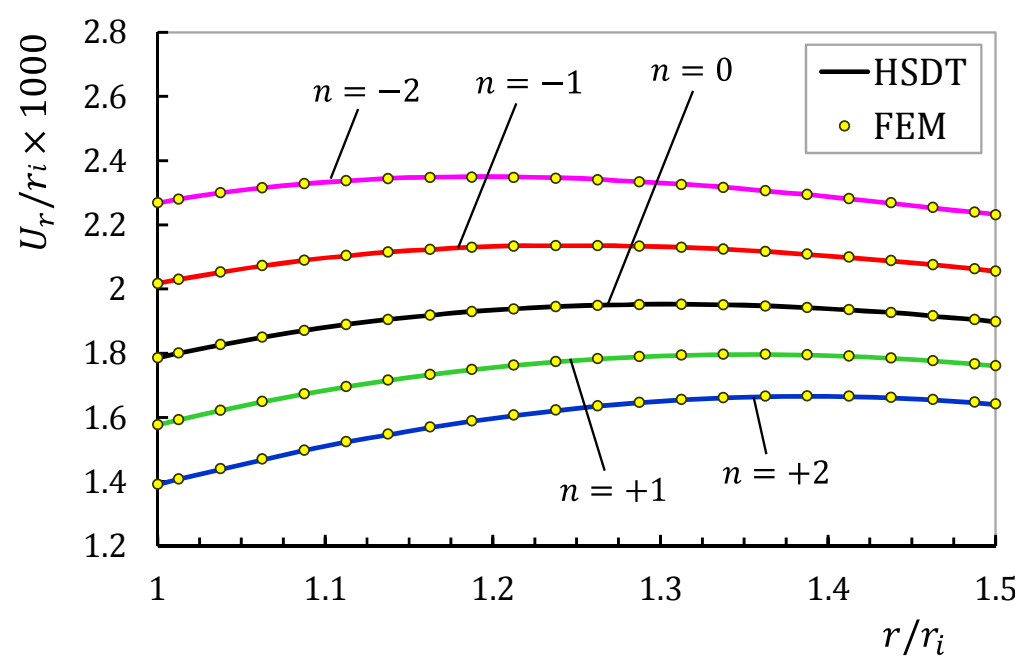

Figure 4: Dimensionless radial displacement distribution in the middle of the cylinder under internal pressure and temperature gradient $\left(\Delta T=100^{\circ} \mathrm{C}\right)$.

Figures 7 and 8 show the distribution of the dimensionless circumferential stress resulting from the numerical and analytical solution at $x=L / 2$ for $100{ }^{\circ} \mathrm{C}$ temperature gradient under pressurized and non-pressurized cylinder, respectively. For both loadings, the circumferential stress for negative values of $n$ is higher than the homogenous materials at layers close to the internal surface while at the outer surface, reverse holds true. For positive values of $n$, the reverse holds true, suggesting that the heterogonous materials have less values of circumferential stress than the homogenous ones at inner surfaces and the higher values of stress at outer surfaces. Considering more uniform stress distribution of the layers and less maximum values of stress for $n<0$ under combined loading, it is interesting to use FG materials with negative values of $n$. Internal pressure causes an increase in the values of circumferential stresses, which would result in positive values of stresses around the internal layer.

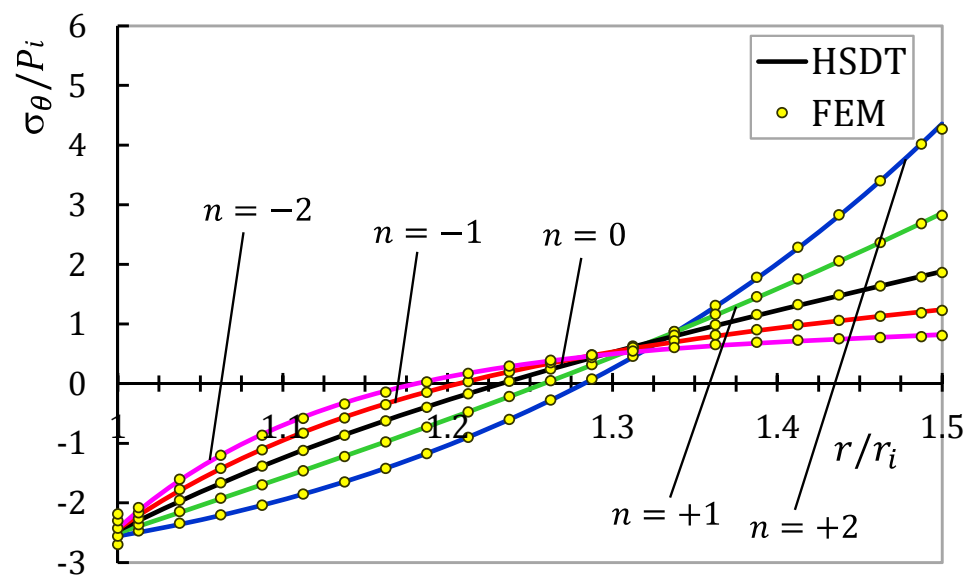

Figure 5: Dimensionless circumferential stress distribution in the middle of the cylinder under temperature gradient $\left(\Delta T=100^{\circ} \mathrm{C}\right)$. 


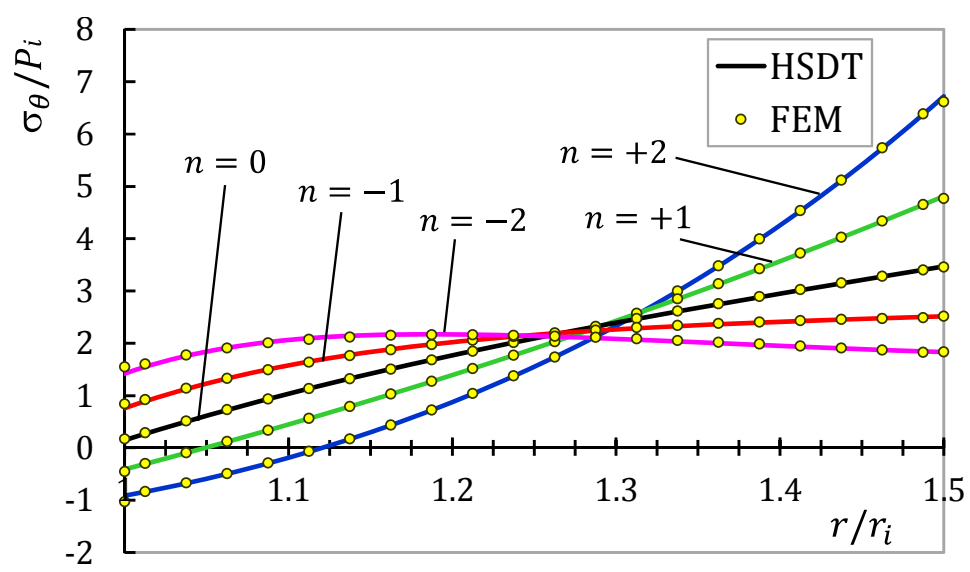

Figure 6: Dimensionless circumferential stress distribution in the middle of the cylinder under internal pressure and temperature gradient $\left(\Delta T=100{ }^{\circ} \mathrm{C}\right)$.

Distribution of von Mises stress resulting from numerical and analytical solution in the middle of the cylinder for thermal loading $\left(\Delta T=100^{\circ} \mathrm{C}\right)$ is illustrated in Figure 7 . It can be seen that von Mises stresses at internal layer are equal for different inhomogeneities and for negative ones a decrease in the value of stresses appears along the thickness relative to external layer. $n>0$ causes higher values of von Mises stresses relative to the homogenous materials while for $n<0$ the reverse hold true. Considering temperature gradient, von Mises stress at the internal half of the cylinder decreases along the thickness from the internal layer to the external one for all inhomogenous materials, while it increases for positive values of $n$ and is constant for negative ones at the external half.

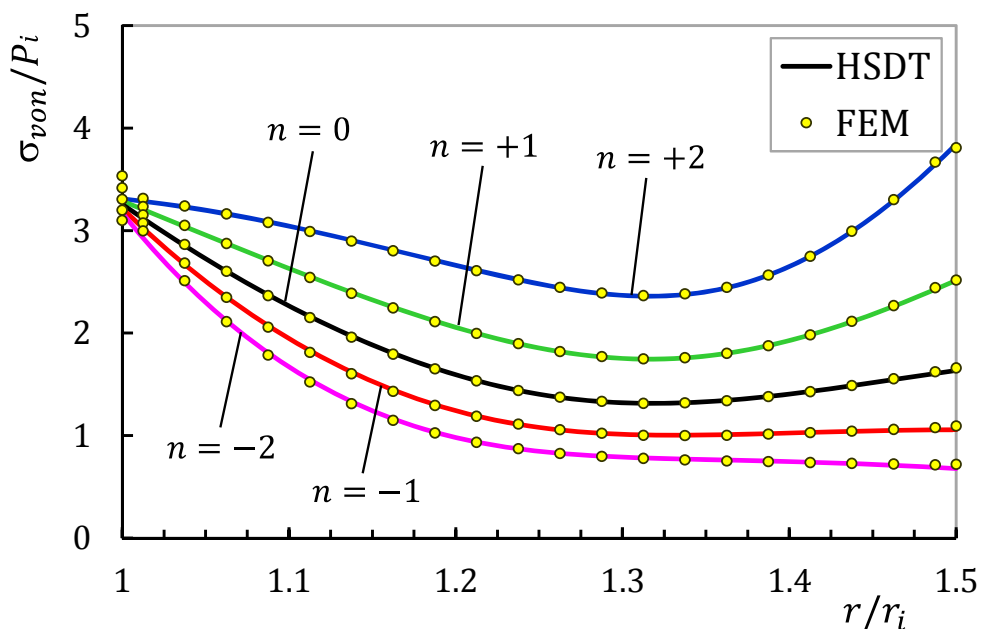

Figure 7: Dimensionless von Mises stress distribution in the middle of the cylinder under temperature gradient $\left(\Delta T=100{ }^{\circ} \mathrm{C}\right)$. 
Figure 8 shows the numerical and analytical distribution of von Mises stress under combined loading at $x=L / 2$. The comparison of Figures 7 and 8 clearly shows that adding internal pressure to the temperature gradient results in different values of von Mises stress at the internal layer, i.e. around the internal layer. Corresponding stress of negative inhomogeneous materials has maximum values while around the external layer, materials with positive inhomogeneities have maximum values. Applying internal pressure to the cylinder under temperature gradient causes less values of von Mises stress around the internal layer and more values around the external one for $n>0$. It could easily be seen that FGMs with negative values of $n$ are more appropriate from the viewpoint of less von Mises stress.

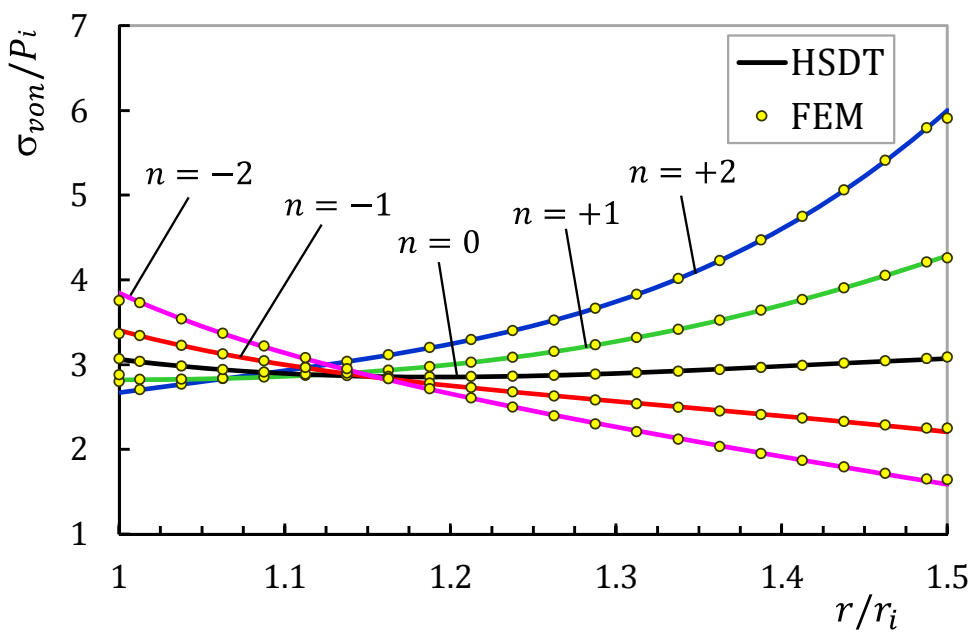

Figure 8: Dimensionless von Mises stress distribution in the middle of the cylinder under internal pressure and temperature gradient $\left(\Delta T=100{ }^{\circ} \mathrm{C}\right)$.

Figure 9 shows the distribution of shear stress resulting from analytical solution along the longitude of cylinder under combined load for $n=+1$ in different layers. It can be seen that there are shear stresses near the two ends of the cylinder under the effect of clamped boundary conditions, which has been considered in shear deformation theory. Dimensionless radial displacement and von Mises stress distribution under combined load at $z=h / 2$ for different materials are illustrated in Figures 10 and 11, respectively. It could be observed that displacement and stress along the longitudinal direction are uniform except the layers around the clamped boundaries. Therefore, PET results are valid through the length of the cylinder far away from clamped boundaries. 


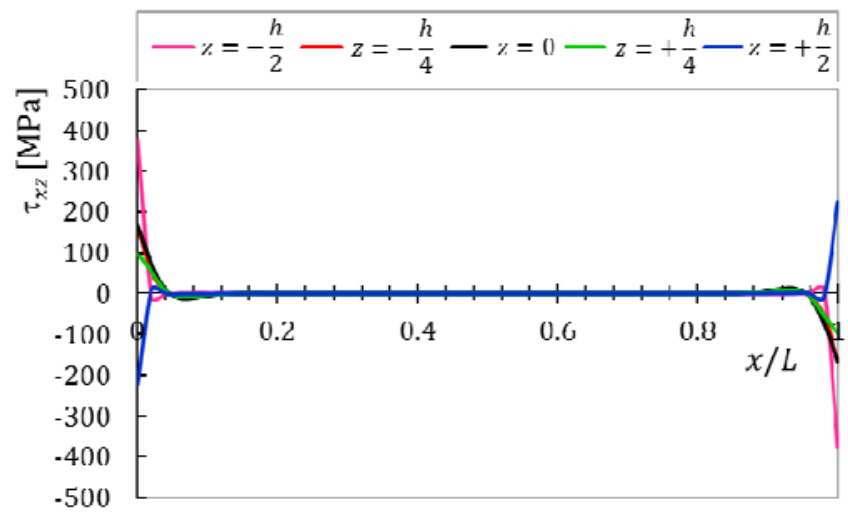

Figure 9: Shear stress distribution in different layers under internal pressure and temperature gradient $\left(\Delta T=100^{\circ} \mathrm{C}\right)$ for $n=+1$.

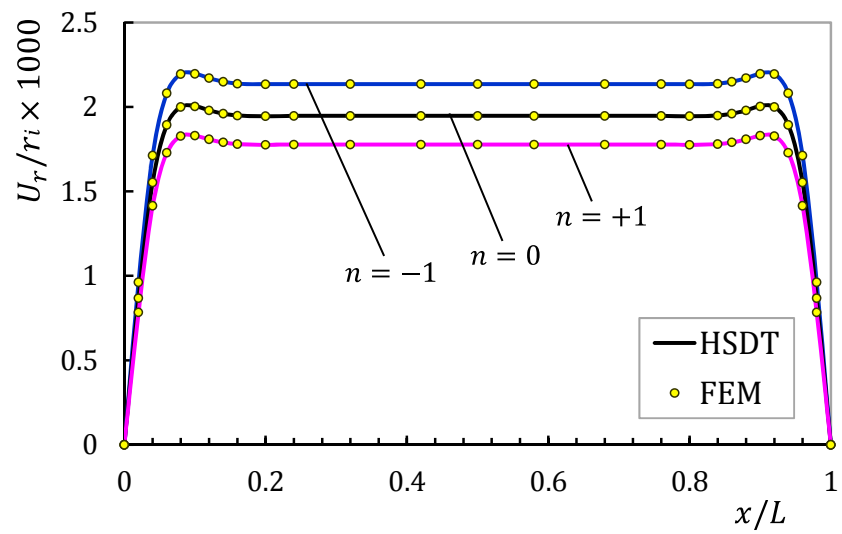

Figure 10: Dimensionless radial displacement distribution at $z=h / 2$ under internal pressure and temperature gradient $\left(\Delta T=100^{\circ} \mathrm{C}\right)$.

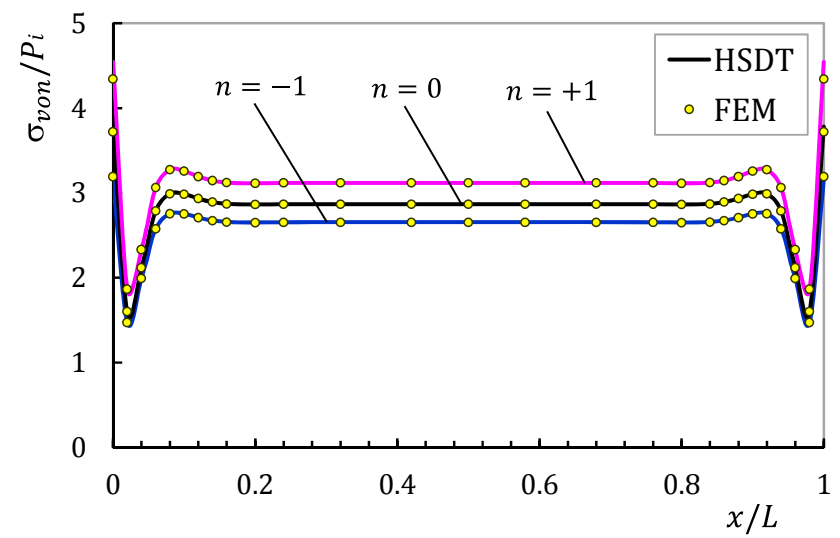

Figure 11: Dimensionless von Mises stress distribution at $z=h / 2$ under internal pressure and temperature gradient $\left(\Delta T=100^{\circ} \mathrm{C}\right)$. 


\subsection{Temperature Gradient Effect}

The effect of temperature gradient on radial displacement for $n=+1$ has been investigated in Figure 12. It could be observed that for $n=-1$, the same graph is obtained. It can be seen that increasing temperature gradient leads to increment in values of radial displacements. This increment is equal from the internal layer to the external one for lower gradient temperature while it is higher in the external layers for upper gradient temperature for inhomogenous materials.

The effect of temperature gradient on von Mises stress has been investigated in Figures 13 and 14 for $n= \pm 1$. The von Mises stresses, as the radial displacements, for both positive and negative values of $n$ are uniform through the thickness in lower temperature gradient. The increment of temperature gradient yields higher stress values. Upper temperature gradient has brought about higher values of stresses around the internal and external layers and low levels of stresses around the middle layer for $n=+1$ while it causes reductions in stresses from the internal to the external layer for $n=-1$. Therefore, the increment of temperature gradient yields higher displacement and stress along the thickness of the cylinder.

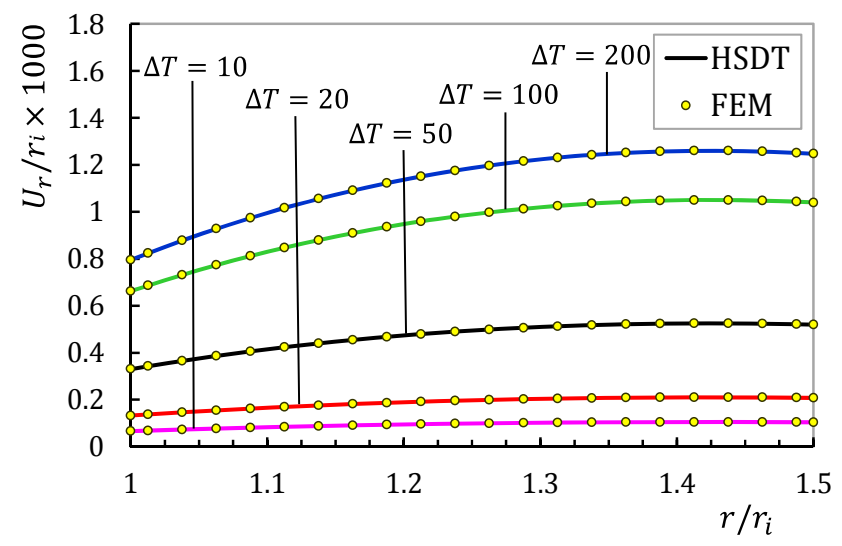

Figure 12: Dimensionless radial displacement distribution in the middle of the cylinder under different temperature gradient for $n=+1$.

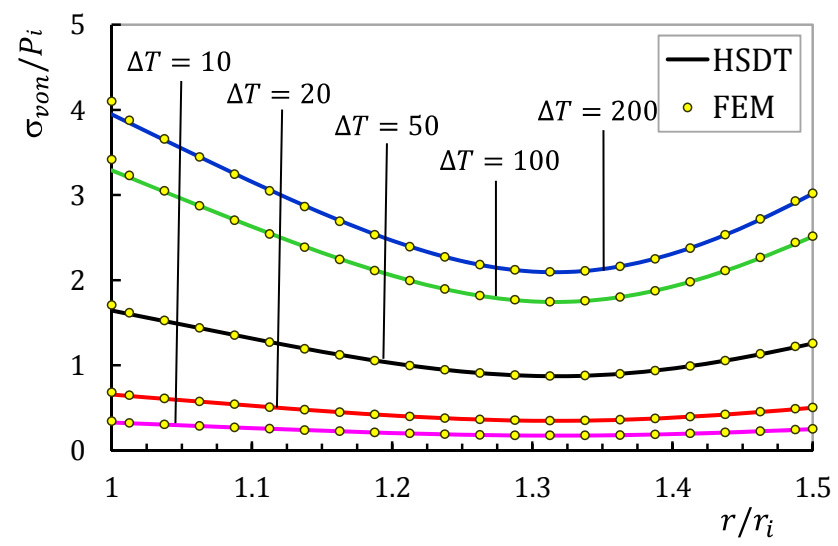

Figure 13: Dimensionless von Mises stress distribution in the middle of the cylinder under different temperature gradient for $n=+1$. 


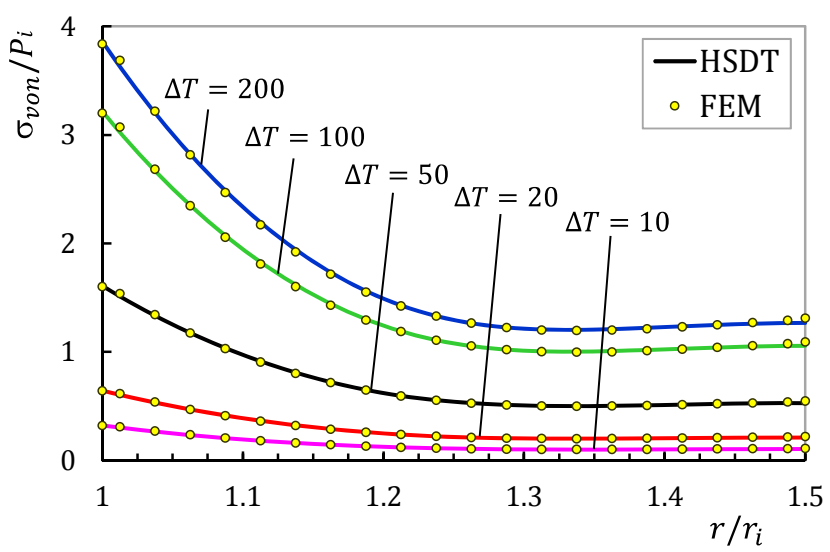

Figure 14: Dimensionless von Mises stress distribution in the middle of the cylinder under different temperature gradient for $n=-1$.

\subsection{Third-order Approximation Effect}

Tables 1 and 2 present the radial and von Mises stresses of different layers resulting from different solutions in the middle of heterogeneous cylinder $(x=L / 2)$ under internal pressure, respectively. The radial and von Mises stresses of different layers resulting from different solutions in the middle of the heterogeneous cylinder $(x=L / 2)$ under $100^{\circ} \mathrm{C}$ temperature gradient are presented in Tables 3 and 4, respectively. The stresses resulting from internal pressure increase from the external layer to the internal one whereas FG materials with positive inhomogeneities yield lower values of the maximum stresses. It has been shown that FSDT method has acceptable results for displacements, but the radial and von Mises stresses resulting from FSDT show a significant difference once compared with the results calculated from HSDT and FEM solution. This difference increases at layers close to the boundaries and the greatest difference occurs in the internal surface $(z=-h / 2)$ while using HSDT yields the values of -80 and 0 for radial stresses at the internal and external layer, respectively, which is compatible with applying pressure. In the cylinder under temperature gradient, the radial stresses at different layers have negative values which are inconsiderable relative to the stresses of pressurized cylinder.

\begin{tabular}{|c|c|c|c|c|c|c|c|c|c|}
\hline \multirow{2}{*}{$\sigma_{r}, \mathrm{MPa}$} & \multicolumn{3}{|c|}{$n=-1$} & \multicolumn{3}{|c|}{$n=0$} & \multicolumn{3}{|c|}{$n=+1$} \\
\hline & HSDT & FSDT & FEM & HSDT & FSDT & FEM & HSDT & FSDT & FEM \\
\hline$z=-h / 2$ & -77.751 & 7.228 & -80.000 & -77.211 & 6.738 & -80.000 & -76.347 & 6.114 & -80.000 \\
\hline$z=-h / 4$ & -47.322 & -14.019 & -46.514 & -50.836 & -12.349 & -49.782 & -54.364 & -10.700 & -52.920 \\
\hline$z=0$ & -24.759 & -27.337 & -24.826 & -28.158 & -27.619 & -28.164 & -31.723 & -27.516 & -31.610 \\
\hline$z=h / 4$ & -9.633 & -35.801 & -10.198 & -11.321 & -40.112 & -12.168 & -13.014 & -44.331 & -14.333 \\
\hline$z=h / 2$ & -1.135 & -41.180 & 0.000 & -1.754 & -50.523 & 0.000 & -2.824 & -61.146 & 0.000 \\
\hline
\end{tabular}

Table 1: Radial stress of different layers under internal pressure at $x=L / 2$ based on SDT and FEM. 


\begin{tabular}{|c|c|c|c|c|c|c|c|c|c|}
\hline \multirow{2}{*}{$\sigma_{V o n}, \mathrm{MPa}$} & \multicolumn{3}{|c|}{$n=-1$} & \multicolumn{3}{|c|}{$n=0$} & \multicolumn{3}{|c|}{$n=+1$} \\
\hline & HSDT & FSDT & FEM & HSDT & FSDT & FEM & HSDT & FSDT & FEM \\
\hline$z=-h / 2$ & 291.64 & 246.61 & 289.50 & 249.67 & 204.72 & 250.90 & 212.26 & 167.66 & 216.12 \\
\hline$z=-h / 4$ & 209.60 & 193.12 & 209.27 & 199.50 & 180.35 & 199.01 & 188.01 & 166.16 & 187.31 \\
\hline$Z=0$ & 154.72 & 155.25 & 154.84 & 161.98 & 161.09 & 162.04 & 167.47 & 164.90 & 167.48 \\
\hline$z=h / 4$ & 117.48 & 127.53 & 117.73 & 134.46 & 145.56 & 134.84 & 151.79 & 163.89 & 152.36 \\
\hline$z=h / 2$ & 92.090 & 93.477 & 92.412 & 115.00 & 132.83 & 114.31 & 141.79 & 163.14 & 139.48 \\
\hline
\end{tabular}

Table 2: von Mises stress of different layers under internal pressure at $x=L / 2$ based on SDT and FEM.

\begin{tabular}{|c|c|c|c|c|c|c|c|c|c|}
\hline \multirow{2}{*}{$\sigma_{r}, \mathrm{MPa}$} & \multicolumn{3}{|c|}{$n=-1$} & \multicolumn{3}{|c|}{$n=0$} & \multicolumn{3}{|c|}{$n=+1$} \\
\hline & HSDT & FSDT & FEM & HSDT & FSDT & FEM & HSDT & FSDT & FEM \\
\hline$z=-h / 2$ & -10.428 & 247.200 & 0.000 & -6.941 & 248.026 & 0.000 & -5.055 & 249.363 & 0.000 \\
\hline$z=-h / 4$ & -9.267 & 216.230 & -13.032 & -12.319 & 244.071 & -14.964 & -15.101 & 276.114 & -17.096 \\
\hline$z=0$ & -13.412 & 192.083 & -13.109 & -16.728 & 240.910 & -16.742 & -21.064 & 302.866 & -21.228 \\
\hline$z=h / 4$ & -10.237 & 172.744 & -7.630 & -12.852 & 238.319 & -10.720 & -16.786 & 329.617 & -14.973 \\
\hline$z=h / 2$ & 5.263 & 156.915 & 0.000 & 4.440 & 236.161 & 0.000 & 3.892 & 356.369 & 0.000 \\
\hline
\end{tabular}

Table 3: Radial stress of different layers under $100{ }^{\circ} \mathrm{C}$ temperature gradient at $x=L / 2$ based on SDT and FEM.

\begin{tabular}{|c|c|c|c|c|c|c|c|c|c|}
\hline \multirow{2}{*}{$\sigma_{\text {Von }}, M P a$} & \multicolumn{3}{|c|}{$n=-1$} & \multicolumn{3}{|c|}{$n=0$} & \multicolumn{3}{|c|}{$n=+1$} \\
\hline & HSDT & FSDT & FEM & HSDT & FSDT & FEM & HSDT & FSDT & FEM \\
\hline$z=-h / 2$ & 257.43 & 118.58 & 255.81 & 260.66 & 118.97 & 264.34 & 263.22 & 119.31 & 273.28 \\
\hline$z=-h / 4$ & 137.60 & 101.86 & 136.02 & 165.15 & 114.97 & 163.95 & 197.88 & 129.77 & 196.94 \\
\hline$z=0$ & 86.270 & 89.181 & 86.172 & 112.19 & 111.84 & 112.11 & 148.31 & 140.32 & 148.24 \\
\hline$z=h / 4$ & 81.040 & 79.259 & 80.329 & 108.86 & 109.34 & 108.41 & 146.90 & 150.94 & 146.70 \\
\hline$z=h / 2$ & 84.500 & 71.294 & 87.278 & 130.83 & 107.29 & 132.63 & 201.17 & 161.62 & 201.17 \\
\hline
\end{tabular}

Table 4: von Mises stress of different layers under $100{ }^{\circ} \mathrm{C}$ temperature gradient at $x=L / 2$ based on SDT and FEM.

It is generally observed that modifying initial approximation of displacement field components in comparison with FSDT, HSDT yields error reduction. The reason is that FSDT assumes linear distribution for the radial displacement while according to the Lame's theory (PET), the variation of radial displacement along the thickness of the cylinder has hyperbolic distribution.

$$
U_{r}(r)=C_{1} r+\frac{C_{2}}{r}
$$

Figure 15 illustrates the effect of third and first-order shear deformation theories in the middle of the cylinder under temperature gradient $\Delta T$ and internal pressure $\left(P_{i}\right)$ for $n=+1$. It could easi- 
ly be seen that around the middle layer, lower approximation has acceptable results. However, around the internal and external layers, FSDT yields different distributions resulting from Eq. (47). This phenomenon intensifies for thermal loading because displacement distribution along the thickness changes from linear status to nonlinear one. The polynomial distribution of displacement in third-order approximation improves the accuracy of the results. The calculus of stresses from displacements and strains by using constitutive relations intensifies the initial error appearing in displacements.

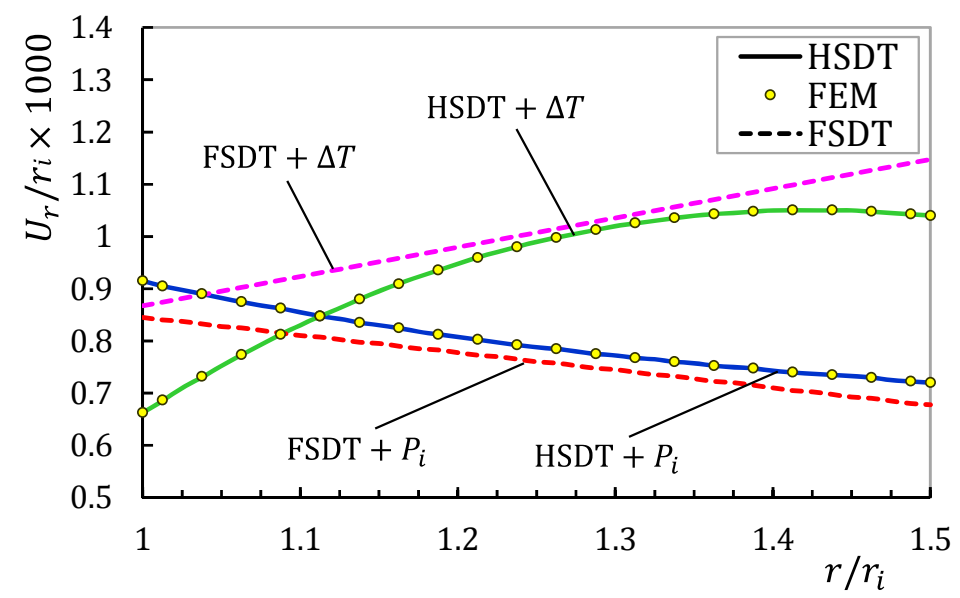

Figure 15: Dimensionless radial displacement distribution in the middle of the cylinder based on HSDT, FSDT and FEM for $n=+1$.

\section{CONCLUSIONS}

In this study, the heterogeneous hollow cylinders with power-law varying elastic modulus along radial direction have been solved by HSDT and FEM, and have been compared with homogenous cylinders. At the boundary areas of a thick-walled cylinder with clamped-clamped ends under thermal and mechanical load, use cannot be made of PET, and SDT must be used instead. The shear stress in boundary areas cannot be ignored, but in areas further away from the boundaries, it could be. Therefore, the non-uniform displacements and stresses at points close to the boundaries are different from the other areas under the effect of shear stresses resulting from boundary conditions and, thus, the PET can be used, provided that the shear strain is zero. It was observed that using negative inhomogeneities in the cylinder causes small decreases under thermal load and considerable increase under mechanical load in radial displacements while the positive inhomogeneities are appropriate under combined loading since less radial displacements appear in the cylinder. In general, the effect of inhomogeneity constants on displacement distribution along the thickness for different temperature gradients is inconsiderable compared with internal pressure. It is observed that the stresses are the function of longitudinal and radial direction. Because of low level and small variation of circumferential stresses, using materials with negative inhomogeneities is more suitable. Unlike the displacements, von Mises stresses are strongly affected by the temperature gradient variations and inhomogeneity constants. Using materials with negative $n$ for the cylinder under thermal or combined loading causes less von Mises stresses. Furthermore, temperature gradient increment 
causes the external layer to be critical from the viewpoint of displacement and internal layer from the viewpoint of stress. It can be concluded that using FSDT for thermoelastic analysis of cylindrical shells, because of disregarding third approximation terms in displacement field components, is not appropriate. Therefore, third-order approximation yields improved accuracy, especially around the boundary layers. Because the terms resulting from pressure and temperature gradient have been revealed in the non-homogeneity part of the set of governing differential equations, the superposition principle could be utilized for the effect of combined loading on the basis of linear elasticity. In fact, the non-homogeneity vector $\{F\}$ appears as a force vector in the governing equations.

\section{References}

Arani, A.G., Kolahchi, R., Barzoki, A.A.M. (2011). Effect of material in-homogeneity on electro-thermo-mechanical behaviors of functionally graded piezoelectric rotating shaft. Applied Mathematical Modelling, 35, 2771-2789.

Arefi, M., Rahimi, G.H. (2010). Thermo elastic analysis of a functionally graded cylinder under internal pressure using first order shear deformation theory. Scientific Research and Essays, 5, 1442-1454.

Cao, L.-L., Qin, Q.-H., Zhao, N. (2012). Hybrid graded element model for transient heat conduction in functionally graded materials. Acta Mechanica Sinica, 28, 128-139.

Eipakchi, H.R. (2010). Third-order shear deformation theory for stress analysis of a thick conical shell under pressure, J. of Mechanics of materials and structures 5(1): 1-17.

Eipakchi, H.R., Khadem, S.E., Rahimi, G.H. (2008). Axisymmetric stress analysis of a thick conical shell with varying thickness under nonuniform internal pressure, J. Engineering Mechanics 134: 601-610.

Eipakchi, H.R., Rahimi, G.H., Khadem, S.E. (2003). Closed form solution for displacements of thick cylinders with varying thickness subjected to nonuniform internal pressure, J. Structural Engineering and Mechanics 16(6): 731-748.

Fukui, Y., Yamanaka, N. (1992). Elastic analysis for thick-walled tubes of functionally graded materials subjected to internal pressure, JSME, Ser. I 35(4): 891-900.

Ghannad, M., \& Gharooni, H. (2015). Elastic analysis of pressurized thick FGM cylinders with exponential variation of material properties using TSDT. Latin American Journal of Solids and Structures, 12(6), 1024-1041.

Ghannad, M., Nejad, M.Z. (2010). Elastic analysis of pressurized thick hollow cylindrical shells with clampedclamped ends, Mechanika 5(85): 11-18.

Ghannad, M., Rahimi, G.H., Nejad, M. Z. (2012). Determination of displacements and stresses in pressurized thick cylindrical shells with variable thickness using perturbation technique. Mechanika, 18(1), 14-21.

Ghannad, M., Rahimi, G.H., Nejad, M.Z. (2013). Elastic analysis of pressurized thick cylindrical shells with variable thickness made of functionally graded materials, Composites: Part B 45: 388-396.

Jabbari, M., Meshkini, M., Eslami, M.R. (2011). Mechanical and thermal stresses in a FGPM hollow cylinder due to radially symmetric loads. ISRN Mechanical Engineering, 2011, Article ID 291409, 1-9. doi:10.5402/2011/291409

Keles, I., Conker, C. (2011). Transient hyperbolic heat conduction in thick-walled FGM cylinders and spheres with exponentially-varying properties. European Journal of Mechanics A-Solids, 30, 449-455.

Koizumi, M. (1993). The concept of FGM: ceramic transactions, Functionally Graded Materials. 34, 3-10.

Nejad, M.Z., Jabbari, M., Ghannad, M. (2015). Elastic analysis of FGM rotating thick truncated conical shells with axially-varying properties under non-uniform pressure loading. Composite Structures, 122, 561-569.

Nejad, M.Z., Rahimi, G.H., Ghannad, M. (2009). Set of field equations for thick shell of revolution made of functionally graded materials in curvilinear coordinate system, Mechanika 3(77): 18-26.

Reddy, J.N., Liu, C.F. (1985). A higher-order shear deformation theory of laminated elastic shells, International J. of Engineering Science 23: 319-330. 
Shao, Z.S., Ma, G.W. (2008). Thermo-mechanical stresses in functionally graded circular hollow cylinder with linearly increasing boundary temperature. Composite Structures, 83, 259-265.

Shi, Z., Zhang, T., Xiang, H. (2007). Exact solutions of heterogeneous elastic hollow cylinders. Composite Structures, $79,140-147$.

Tutuncu, N. (2007). Stresses in thick-walled FGM cylinders with exponentially-varying properties, J. Engineering Structures 29: 2032-2035.

Xiang, H., Shi, Z., Zhang, T. (2006). Elastic analyses of heterogeneous hollow cylinders. Mechanics Research Communications, 33, 681-691.

Xue, C.X., Pan, E. (2013). On the longitudinal wave along a functionally graded magneto-electro-elastic rod. International Journal of Engineering Science, 62, 48-55.

Yamanoushi, M., Koizumi, M., Hiraii, T., Shiota, I., Eds. (1990). Proceedings of the 1st International Symposium on Functionally Gradient Materials. Sendai, Japan, 273-281.

Zafarmand, H., Salehi, M., Asemi, K. (2015). Three dimensional free vibration and transient analysis of two directional functionally graded thick cylindrical panels under impact loading. Latin American Journal of Solids and Structures, 12(2), 205-225.

\section{Appendices}

$$
\begin{aligned}
& {[A]=\left[\begin{array}{ll}
{\left[A_{11}\right]_{4 * 4}} & {\left[A_{12}\right]_{4 * 4}} \\
{\left[A_{21}\right]_{4 * 4}} & {\left[A_{22}\right]_{4 * 4}}
\end{array}\right]} \\
& {\left[A_{12}\right]=\left[A_{21}\right]=0} \\
& {\left[A_{11}\right]_{i j}=\left\{\begin{array}{lc}
R \lambda^{\prime} \int_{-h / 2}^{h / 2} e^{n(\bar{r}-1)}(1-v)\left(1+\frac{z}{R}\right) z^{(i+j-2)} d z & i, j=2,3,4 \\
0 & \text { else }
\end{array}\right.} \\
& {\left[A_{22}\right]_{i j}=\left\{R \lambda^{\prime} \int_{-h / 2}^{h / 2} k e^{n(\bar{r}-1)}\left(1+\frac{Z}{R}\right) z^{(i+j-2)} d z \quad i, j=1,2,3,4\right.} \\
& {[B]=\left[\begin{array}{ll}
{\left[B_{11}\right]_{4^{* 4}}} & {\left[B_{12}\right]_{4^{* 4}}} \\
{\left[B_{21}\right]_{4^{* 4}}} & {\left[B_{22}\right]_{4^{* 4}}}
\end{array}\right]} \\
& {\left[B_{22}\right]=0} \\
& {\left[B_{21}\right]=-\left[B_{12}\right]^{T}} \\
& {\left[B_{11}\right]_{i j}=\left\{\begin{array}{cc}
R \lambda^{\prime} \int_{-h / 2}^{h / 2} e^{n(\bar{r}-1)}(1-v)\left(1+\frac{z}{R}\right) z^{(i+j-2)} d z & i=1, j=2,3,4 \\
0 & j=1, i=2,3,4
\end{array}\right.}
\end{aligned}
$$




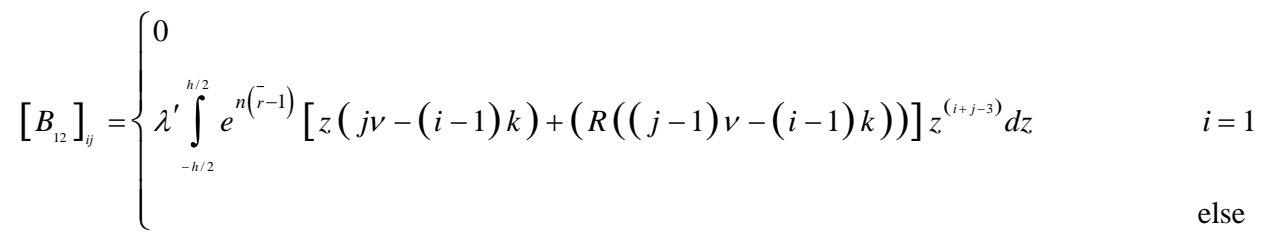

$$
\begin{aligned}
& {[C]=\left[\begin{array}{ll}
{\left[C_{11}\right]_{4 * 4}} & {\left[C_{12}\right]_{4 * 4}} \\
{\left[C_{21}\right]_{4 * 4}} & {\left[C_{22}\right]_{4 * 4}}
\end{array}\right]} \\
& {\left[C_{21}\right]=-\left[C_{12}\right]^{T}} \\
& {\left[C_{11}\right]_{i j}=\left\{\begin{array}{l}
R \lambda^{\prime} \int_{-h / 2}^{h / 2} e^{n(\bar{r}-1)}(1-v)\left(1+\frac{Z}{R}\right) d z \\
-(i-1)(j-1) R \lambda^{\prime} k \int_{-h / 2}^{h / 2} e^{n(\bar{r}-1)}\left(1+\frac{Z}{R}\right) z^{(i+j-4)} d z
\end{array}\right.} \\
& {\left[C_{12}\right]_{i j}=\left\{\begin{array}{lc}
\lambda^{\prime} \int_{-h / 2}^{h / 2} v e^{n(\bar{r}-1)} d z & i, j=1 \\
\lambda^{\prime} \int_{-h / 2}^{h / 2} v e^{n(\bar{r}-1)} z^{j-2}((j-1) R+j z) d z & \text { else } \\
0 & i=1, j=2,3,4
\end{array}\right.} \\
& {\left[C_{22}\right]_{i j}=\left\{\begin{array}{c}
-\lambda^{\prime} \int_{-h / 2}^{h / 2} e^{n(\bar{r}-1)}\left[((i-1)(j-1)(R+z)(1-v))+((i+j-2) v) z+\left(\frac{1-v}{R+z}\right) z^{2}\right] z^{(i+j-4)} d z \\
i, j=1,2,3,4
\end{array}\right.} \\
& i, j=1 \\
& \text { else }
\end{aligned}
$$

where the parameters $\lambda^{\prime}$ and $k$ are as follows

$$
\begin{gathered}
\lambda^{\prime}=\frac{E_{i}}{(1+v)(1-2 v)} \\
k=\left(\frac{1-2 v}{2}\right)
\end{gathered}
$$

